T. Lehrnbecher ${ }^{1}$ H.-J. Laws ${ }^{2}$

\section{Infektionen in der pädiatrischen Hämatologie und Onkologie}

\author{
Infectious Complications in Pediatric Cancer Patients
}

\section{Zusammenfassung}

Trotz aller Fortschritte in der Supportivtherapie gehören Infektionen immer noch zu häufigen und potenziell lebensbedrohlichen Komplikationen bei krebskranken Kindern. Die Granulozytopenie ist der wichtigste Einzelrisikofaktor für Infektionen, jedoch bedingt die Therapie der Krebserkrankung eine komplexe Störung aller Arme des Immunsystems und macht die Kinder für eine Vielzahl von opportunistischen Keimen empfänglich. Heutzutage werden bei krebskranken Kindern überwiegend Grampositive Erreger isoliert, aber Gramnegative Erreger können binnen kürzester Zeit zu schwersten septischen Krankheitsbilden führen. Bei prolongierter Granulozytopenie steigt zudem das Risiko für eine Pilzinfektion. Während Fieber oft das einzige Zeichen einer Infektion ist, fehlen gerade in der Frühphase der Infektion meist spezifische klinische oder radiologische Zeichen. Da auch keine Laborparameter existieren, die frühzeitig und verlässlich eine Infektion anzeigen, erhalten heute alle Kinder mit Fieber bei Granulozytopenie eine empirische antibiotische Therapie. Eine Stratifizierung durch klinische oder genetische Risikofaktoren wird derzeit evaluiert, kann aber noch nicht allgemein empfohlen werden. Möglicherweise lässt sich hierdurch die Intensität der antimikrobiellen Prophylaxe entsprechend des individuellen Risikos des Patienten steuern sowie nur die Patienten, die tatsächlich Infektionen haben, rasch einer suffizienten und individuell angepassten Therapie zuführen.

\section{Schlüsselwörter}

Krebserkrankungen · Kinder · Infektionen · Erreger · Risikofaktoren

\section{Abstract}

Infectious complications are still a major cause of morbidity and mortality in pediatric patients undergoing therapy for malignancy. Therapy-induced neutropenia is the most important risk factor for infectious risk in pediatric patients with cancer, but other factors, such as alterations in skin/mucosal barriers, and defects in cell-mediated or humoral immunity also contribute to the risk for infection. In most centers, about two thirds of bacteremic isolates are gram-positive pathogens, whereas gramnegative organisms are isolated less frequently, but are associated with considerably higher mortality rates. Prolonged neutropenia increases the risk for invasive fungal infection. In most cases, fever is an important and early indication of serious infection, particularly in children with neutropenia. Discrimination between serious and inconsequential infection in febrile children with neutropenia at the time of presentation is difficult, and serum markers have not been proven to reliably indicate infection. Although several groups investigate risk categories based on clinical tests or the genetic background, the current paradigm is to treat all pediatric patients with neutropenia and fever with intravenous broad-spectrum antibiotics. It is hoped that the identification of one or more predictive factors may be useful for tailoring antibiotic prophylaxis and therapy in children with cancer.

\section{Key words}

Cancer $\cdot$ child $\cdot$ infectious complication $\cdot$ pathogen $\cdot$ risk factor

${ }^{1}$ Pädiatrische Hämatologie und Onkologie, Universität Frankfurt ${ }^{2}$ Klinik für Kinder-Onkologie, -Hämatologie und -Immunologie, Universitätsklinikum Düsseldorf 
Infektionen als Ursache für Morbidität und Mortalität

Mit immer intensiveren Therapiemodalitäten versucht man die Heilungsrate bei Kindern mit Krebserkrankungen zu verbessern. Durch die Therapieintensivierung wird jedoch eine zunehmende Immunsuppression hervorgerufen, so dass infektiöse Komplikationen trotz Verbesserungen in der Supportivtherapie noch immer eine wichtige Ursache für Morbidität und Mortalität bei Kindern mit Krebserkrankungen sind [9, 10, 41]. Infektionen treten bei Kindern mit akuter lymphatischer Leukämie nach etwa einem Viertel der Chemotherapiezyklen auf [25], während Kinder mit akuter myeloischer Leukämie, die eine deutlich intensivere Chemotherapie erhalten, nach mehr als $80 \%$ der Chemotherapieeinheiten zum Teil sehr schwer verlaufende infektiöse Komplikationen erleiden [22]. Die im Rahmen von Infektionen notwendigen diagnostischen und therapeutischen Maßnahmen sind kostspielig und bedeuten für die Patienten eine weitere Einschränkung der Lebensqualität. Zudem birgt die antibiotische Therapie neben potenziellen Medikamentennebenwirkungen die Gefahr von Resistenzentwicklungen. Zusätzliche Krankenhausaufenthalte belasten den Patienten und dessen Familie und erhöhen das Risiko einer schwer therapierbaren nosokomialen Infektion. Trotz potenter Antibiotika ist jede infektiöse Komplikation eine potenzielle Lebensgefahr für die Patienten. So schwankt je nach maligner Grunderkrankung die Infektions-assoziierte Letalität heute zwischen 1 und 6\% [22, 25, 46]. Neben der unmittelbaren Morbidität und Mortalität durch Infektionen müssen wegen infektiöser Komplikationen oft Chemotherapieund Bestrahlungseinheiten unterbrochen oder verschoben werden und verlieren so an Effektivität. Das erhöhte Infektionsrisiko bei Patienten mit Malignom ist einer der entscheidenden Faktoren, die eine Intensivierung dieser Therapie verhindern und somit die Heilungs- und Überlebenschancen dieser Patienten limitieren. Deshalb ist es notwendig, Prophylaxe und Therapie von infektiösen Komplikationen bei Kindern mit Krebserkrankungen zu verbessern. Im Sinne der Bestrebungen der Qualitätssicherung durch die GPOH/KPOH sollen die nachfolgenden Kapitel die derzeitigen Strategien näher beleuchten, grundlegende Probleme aufzeigen und Zukunftsperspektiven darstellen [8].

Die antineoplastische Therapie ruft eine komplexe Störung des Immunsystems hervor

Bei Kindern mit Krebserkrankungen wird sowohl durch die Grunderkrankung selbst als auch durch die notwendige antineoplastische Therapie wie Chemotherapie oder Bestrahlung eine komplexe Störung aller Abwehrsysteme hervorgerufen [21] (Abb.1). Hierbei ist die Granulozytopenie (definiert als absolute

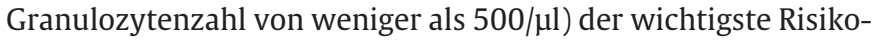
faktor für schwerwiegende bakterielle Infektionen (Tab.1). Bei persistierender Granulozytopenie sind diese Patienten neben bakteriellen Organismen auch von invasiven Hefe- und Schimmelpilzinfektionen bedroht [33]. Dabei haben Patienten, deren Granulozytenwerte im Verlauf einer infektiösen Komplikation ansteigen, eine deutlich bessere Prognose als Patienten, deren Granulozytenzahlen nicht ansteigen oder sogar weiter abfallen $[4,46]$. Auch wenn hämatopoetische Wachstumsfaktoren wie G-CSF oder GM-CSF die Phasen der Granulozytopenie verkürzen, konnte durch sie die Infektions-assoziierte Mortalität bei granulozytopenischen Kindern mit Fieber bzw. dokumentierten Infektionen nicht signifikant vermindert werden [24, 28, 37]. Der positive Effekt von Granulozytentransfusionen bei granulozytopenischen Kindern mit Sepsis und invasiven Pilzinfektionen ist in

\section{"Barrieren" \\ -Verletzung der Haut und \\ Schleimhäute \\ -Veränderungen der endogenen Flora \\ -Zentrale Katheter}

\section{Angeborene Immunität \\ Klonale / erworbene Immunität}

Beeinträchtigung von:

-Komplement

-Akute Phase Proteinen

-Immunregulatoren

(Zytokine, Interferone)

Natürliche Killer Zellen

-Verminderte Zahl von NK Zellen

-Dysfunktion der NK Zellen

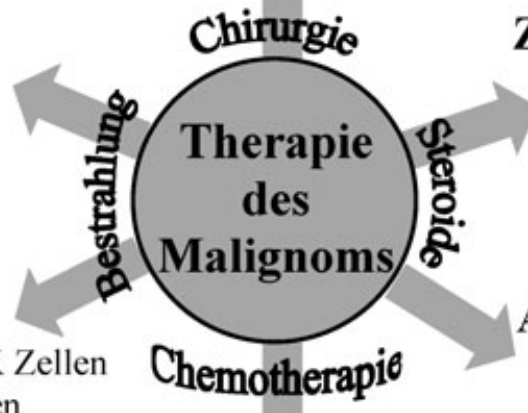

\section{Zellvermittelte Immunität}

-Verminderte Zahl zirkulierender

Lymphozyten

-Imbalance der Lymphozyten-Untergruppen

-Dysfunktion der Lymphozyten

(Signalübertragung)
Antikörper

-Verminderte Zahl von B-Zellen

-Verminderte Produktion von Immunglobulinen

\section{Phagozyten \\ -Verminderte Zahl von zirkulierenden \\ Neutrophilen, Monozyten \\ -Defekte in den Phagozytenfunktionen}

Abb. 1 Komplexe Störung des Immunsystems durch die antineoplastische Therapie (modifiziert nach [21]). 
Tab. 1 Infektionserreger bei krebskranken Kindern und Jugendlichen. Die am häufigsten vorkommenden Erreger sind hervorgehoben.

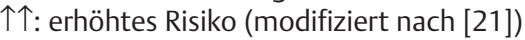

\begin{tabular}{|c|c|c|c|c|c|}
\hline Defekte von & Phagozyten & B-Zellen & T-Zellen & NK-Zellen & Haut/Schleimhaut \\
\hline Staphylokokken & $\uparrow \uparrow$ & & & & $\uparrow \uparrow$ \\
\hline Escherichia coli & $\uparrow \uparrow$ & & & & \\
\hline Klebsiella spp. & $\uparrow \uparrow$ & & & & \\
\hline Enterobacter spp. & $\uparrow \uparrow$ & & & & \\
\hline Pseudomonas aeroginosa & $\uparrow \uparrow$ & & & & \\
\hline Haemophilus influenza Typ B & & $\uparrow \uparrow$ & & & \\
\hline Neisseria meningitidis & & $\uparrow \uparrow$ & & & \\
\hline Salmonella spp. & & $\uparrow \uparrow$ & & & \\
\hline Campylobacter spp. & & $\uparrow \uparrow$ & & & \\
\hline \multicolumn{6}{|l|}{ Viren } \\
\hline Herpes-simplex-Virus & & & $\uparrow \uparrow$ & $\uparrow \uparrow$ & \\
\hline Varizella-zoster-Virus & & & $\uparrow \uparrow$ & & \\
\hline Cytomegalievirus & & & $\uparrow \uparrow$ & $\uparrow \uparrow$ & \\
\hline Epstein-Barr-Virus & & & $\uparrow \uparrow$ & & \\
\hline RS-Virus & & & $\uparrow \uparrow$ & & \\
\hline Adenovirus & & & $\uparrow \uparrow$ & & \\
\hline Influenza & & & $\uparrow \uparrow$ & & \\
\hline \multicolumn{6}{|l|}{ Pilze } \\
\hline Candida spp. & $\uparrow \uparrow$ & & $\uparrow \uparrow$ & & $\uparrow \uparrow$ \\
\hline Toxoplasma gondii & & & $\uparrow \uparrow$ & & \\
\hline
\end{tabular}

Fallberichten und kleinere Serien beschrieben, größere Studien fehlen jedoch [15].

Neben der Granulozytopenie erhöhen jedoch auch therapie-induzierte funktionelle Phagozytendefekte (wie bei Steroidgaben), Störungen der zellulären und humoralen Immunantwort (wie Störungen der T-Zell-Immunität durch Cyclophosphamid oder Purinanaloga sowie Störungen der Immunglobulinsynthese durch Steroide oder Methotrexat), Störungen der Natürlichen Killer (NK) Zellen und des angeborenen Immunsystems sowie Läsionen der Haut und Schleimhäute (durch Katheter, Punktionen, Mukositis) das Risiko für infektiöse Komplikationen [21].

\section{Erregerspektrum}

Durch die Störung der verschiedenen Arme des Immunsystems sind Patienten, die eine zytotoxische Behandlung erhalten, für eine Vielzahl opportunistischer Keime empfänglich (Tab.1). So sind Kinder mit Defekten der körpereigenen Barrieren oder Kinder mit einer Granulozytopenie vor allem durch bakterielle Erreger bedroht. Während in den 1970er-Jahren noch Gramnegative Erreger wie Pseudomonas aeruginosa, Escherichia coli oder Klebsiella pneumoniae vorherrschten und etwa 70\% aller Bakteriämien verursachten [3], werden heute überwiegend Grampositive Erreger gefunden, insbesondere Staphylokokken und Streptokokken [45-47]. Dabei ist der weit verbreitete Einsatz von zentralen Kathetern einer der Hauptgründe für die Zunahme von Koagulase-negativen Staphylokokken, während Infektionen durch Streptokokken der Viridans-Gruppe gehäuft nach Chemotherapieeinheiten gesehen werden, die hochdosiertes CytarabinArabinosid beinhalten. Bei diesen Patienten sind schwerste septische Krankheitsbilder mit Hypotension und pulmonalen Problemen nicht selten [7, 22, 32]. Bedenklich ist die Zunahme von Infektionen durch multiresistente Enterokokken [31], die insbesondere bei einen gehäuften Einsatz von Glycopeptidantibio- 
tika (Vancomycin, Teicoplanin) auftreten [40] (siehe hierzu die Kapitel von Laws et al. sowie von Simon et al. zur Behandlung des Fiebers unklarer Genese bzw. zur Therapie bakterieller Infektionen).

Bei schwerer und prolongierter Granulozytopenie, die durch ab-

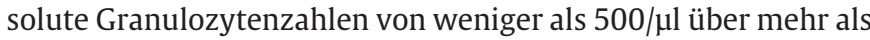
10 Tage definiert ist, wächst mit jedem Tag die Gefahr einer invasiven Pilzinfektion. Die invasiven Pilzinfektionen werden meist durch Candida spp. (insbesondere durch C. albicans) oder Aspergillus spp. verursacht, jedoch werden auch immer häufiger nonalbicans Arten und seltene Pilze wie Mucor, Fusarium oder Trichosporon beschrieben, die auf die herkömmlichen Antimykotika vermindert empfindlich sind [16] (siehe hierzu das Kapitel von Groll und Ritter zur Diagnostik und Therapie von Pilzerkrankungen). Eine eingeschränkte T-Zell-Immunität, wie sie vor allem bei Patienten nach allogenen Stammzelltransplantationen besteht, ist die Ursache für ein deutlich erhöhtes Risiko von invasiven Pilzinfektionen sowie von schweren viralen Infektionen, zum Beispiel durch CMV, HSV, oder Adenoviren [5, 14] (siehe hierzu das Kapitel von Schuster et al. zur Blutstammzelltransplantation).

Allerdings werden nur in etwa einem Drittel der Fälle für eine Infektion ursächliche Erreger nachgewiesen (mikrobiologisch dokumentierte Infektion), während in den übrigen Fällen entweder klinisch eine Infektion ohne Erregernachweis diagnostiziert werden kann (z.B. Pneumonie, Weichteilinfektion; klinisch dokumentierte Infektion) oder lediglich Fieber auf eine mögliche Infektion hinweist (Fieber unbekannter Genese $=$ fever of unknown origin, FUO) [22].

\section{Klinische Zeichen einer Infektion}

Die meisten krebskranken Patienten mit einer therapiebedingten Granulozytopenie entwickeln im Rahmen einer Infektion Fieber, das so als Surrogatmarker für eine Infektion dient. Bei diesen Patienten fehlen insbesondere in der Frühphase einer Infektion spezifische klinische oder radiologische Zeichen, so dass zu diesem Zeitpunkt weder zwischen leicht und schwer verlaufenden Infektionen unterschieden werden kann noch Rückschlüsse auf den Erreger möglich sind. Da bakterielle Infektionen bei abwehrgeschwächten Patienten binnen kürzester Zeit einen nicht mehr beeinflussbaren tödlichen Verlauf nehmen können, ist es heute üblich, alle Kinder mit Granulozytopenie und Fieber stationär mit einer empirischen Antibiotikatherapie intravenös zu behandeln. Je nach Zentrum werden dabei unterschiedliche Definitionen von Fieber verwendet, wobei in den folgenden Kapiteln Fieber als Temperatur von $38,5^{\circ} \mathrm{C}$ und höher bei einmaliger Messung bzw. als Temperatur von 38,0 bis $38,5^{\circ} \mathrm{C}$ über 2 Stunden definiert wird. Dies gilt jeweils nur ohne eine gleichzeitige fiebersenkende Therapie. Umgekehrt ist es notwendig, auch Kinder mit unspezifischen Zeichen oder Symptomen, die auf eine mögliche Infektion hinweisen, ausreichend zu überwachen und gegebenenfalls schnell mit einer antimikrobiellen Therapie zu intervenieren. Mit diesem Vorgehen konnte die früher inakzeptabel hohe Mortalität deutlich gesenkt werden [6]. Allerdings werden auch so Kinder behandelt, die wahrscheinlich keine oder nur eine orale Antibiotikatherapie bräuchten (siehe unten). Es ist wichtig darauf hinzuweisen, dass die Betreuung von immunsupprimierten Patienten mit infektiösen Komplikationen eine Vielzahl von Voraussetzungen erfordert. So sind unter anderem speziell geschultes ärztliches und pflegerisches Personal in einem akzeptablen Personalschlüssel sowie eine pädiatrisch-interdisziplinäre Intensivstation zu fordern. Weiterhin muss die zeitnahe Versorgung mit Blut- und Blutbestandteilen ebenso gewährleistet sein wie die Verfügbarkeit eines Notfalllabors, in dem jederzeit Blutbild, Elektrolyte, Kreatinin, Harnstoff, Leberwerte und eine Gerinnungsanalyse mit Antithrombin III und D-Dimeren bestimmt werden können.

\section{Stratifizierung durch klinische Risikofaktoren}

Patienten mit Fieber bei Granulozytopenie sind eine heterogene Gruppe von Patienten, die jeweils ein unterschiedliches Risiko für schwere Infektionen aufweisen. Entsprechend versuchen verschiedene Arbeitsgruppen, das therapeutische Vorgehen bei Fieber bei Granulozytopenie an das Risiko des individuellen Patienten zu adaptieren. Zwar ist, als Extremfall, die prinzipielle Machbarkeit eines kompletten Verzichtes auf eine antibiotische Therapie bei einer kleinen Gruppe hospitalisierter Kinder mit sehr niedrigem Risiko einer schweren Infektion in einer monozentrischen Studie gezeigt worden [30], jedoch ist dieses Vorgehen bei Kindern von einer breiten klinischen Anwendung weit entfernt. Bei Erwachsenen mit Krebserkrankungen wurden in prospektiven Studien bereits klinische Parameter validiert, durch die Patienten mit sehr niedrigem Risiko für schwere Infektionen identifizieren werden, die dann ambulant und/oder mit oralen Antibiotika behandelt werden können [18, 42-44]. Auch bei Kindern sind in verschiedenen, meist retrospektiven Studien Faktoren beschrieben worden, mit deren Hilfe das Risiko abgeschätzt werden kann, im Verlauf der Infektion schwere Komplikationen zu erleiden. So wurden die Faktoren prolongierte Granulozytopenie [17], niedrige Monozytenzahl bei Aufnahme [2, $27,36]$, hohes Fieber $\left(>39,8^{\circ} \mathrm{C}\right)[17,27,34,36]$ oder Schüttelfrost bei Aufnahme [26], Hypotension oder Schock [17, 26] oder vorherige Episoden von Fieber bei Granulozytopenie (mit oder ohne Erregernachweis) [1] mit einem hohen Risiko für Bakteriämien assoziiert. Die Sensitivität und Spezifität dieser Risikofaktoren müssen jedoch vor einer praktischen Anwendung in prospektiven multizentrischen Studien bestätigt werden. Aus diesem Grunde werden derzeit noch alle Kinder und Jugendlichen mit Fieber bei Granulozytopenie, auch diejenigen mit vermutlich niedrigem Risiko einer schweren Infektion, initial stationär mit intravenösen Antibioika behandelt.

\section{Genetische Disposition}

Warum bei gleicher Grunderkrankung und gleicher Therapie ein Patient schwere infektiöse Komplikationen erleidet und ein anderer nicht, ist immer noch unklar. Es gibt Hinweise, dass kleine genetische Unterschiede, so genannte Polymorphismen, die Inzidenz und den Schweregrad infektiöser Komplikationen bei immunsupprimierten Patienten beeinflussen. Während durch die hohe Redundanz im menschlichen Immunsystem diese Unterschiede in der biologischen Aktivität oder der Serumkonzentration der kodierten Moleküle bei immunkompetenten Individuen 
wahrscheinlich keine oder nur geringe Auswirkungen haben, könnten Polymorphismen gerade bei den Patienten eine große Rolle spielen, bei denen ein oder mehrere Hauptarme des Immunsystems defekt sind. So ist bei Patienten mit Komplementdefekt ein bestimmter Polymorphismus des Fc $\gamma$-RII a mit einem höheren Risiko für eine Meningokokkensepsis assoziiert [12, 35]. Auch erleiden Patienten mit einem Polymorphismus des Fc $\gamma$-RII a nach HLA-identer Knochenmarktransplantation signifikant mehr Infektionen als Patienten, die diesen Polymorphismus nicht aufweisen [38]. Interessanterweise sind Patienten, deren HLA-idente Knochenmark-Spender einen bestimmten Genotyp der in den Phagozyten vorkommenden Myeloperoxidase haben, besonders durch schwere bakterielle Infektionen bedroht [38]. In einer jüngeren Untersuchung konnte gezeigt werden, dass Kinder mit AML, die einen bestimmten Polymorphismus im IL-6Gen oder einen Polymorphismus im Gen der Chitotriosidase aufweisen, ein höheres Risiko für eine Infektion mit Gramnegativen Erregern haben [19].

Untersuchungen zur Assoziation von Polymorphismen von Molekülen des angeborenen Immunsystems mit dem Risiko und dem Verlauf von Infektionen und immunologischen Komplikationen könnten zukünftig zur Erstellung eines „immunologischen Profils" für jeden Patienten mit Immundefekt beitragen, wodurch die Patienten in verschiedene Risikogruppen eingeteilt werden könnten. So ließen sich Vorsorgeprogramme und Therapieregime individuell auf den Patienten abstimmen. Patienten mit hohem Risiko für infektiöse Komplikationen könnten entsprechend intensiver betreut werden, während bei Patienten mit niedrigem Risiko für Komplikationen unnötige Belastungen vermieden würden.

\section{Laborparameter}

Trotz zahlreicher Studien sind bisher keine Laborparameter etabliert, durch die frühzeitig und mit ausreichender Sicherheit granulozytopenische Kinder mit Fieber, die eine schwere Infektion haben und einer intensiven Behandlung bedürfen, von den Kindern unterschieden werden können, die lediglich eine kurzzeitige oder eventuell gar keine antibiotische Therapie benötigen. Das C-reaktive Protein ( $\mathrm{CrP}$ ) wird in vielen Institutionen als Marker für den Schweregrad einer Infektion sowie als Verlaufsparameter während der Therapie verwendet. Da die Konzentrationen des $\mathrm{CrP}$ im Serum verzögert ansteigt, ist der hauptsächliche Informationsgehalt dieses Parameters bei granulozytopenischen Patienten mit Fieber vor allem der negative Vorhersagewert von mindestens zwei Bestimmungen in den ersten 24 Stunden [39]. Dahingegen eignet sich das CrP aufgrund der niedrigen Spezifität und des niedrigen positiven Vorhersagewertes nicht in der Frühdiagnostik bei Patienten mit Granulozytopenie und Fieber $[13,23]$. Für andere Moleküle, wie das Interleukin-6 (IL-6), das Interleukin-8 (IL-8) oder das Procalcitonin, bei denen im Verlauf einer Infektion deutlich früher als bei $\mathrm{CrP}$ ein Konzentrationsanstieg im Serum gemessen werden kann, wird der Wert in der Frühdiagnostik bei granulozytopenischen Kindern mit Fieber derzeit kontrovers beurteilt [11, 13, 20, 29].

\section{Algorithmen der antimikrobiellen Therapie}

Wie in den folgenden Kapiteln weiter erläutert, wird die Entscheidung für den Beginn, für die Modifikation und für die Beendigung einer antimikrobiellen Behandlung von abwehrgeschwächten Kindern entsprechend komplizierter Algorithmen getroffen. Allerdings muss vor einer schematischen Anwendung dieser Algorithmen gewarnt werden, die in keiner Weise die Erfahrung eines spezialisierten Kinderonkologen ersetzen können. Zusätzliche Aspekte, wie zum Beispiel die Möglichkeiten der jeweiligen ambulanten Betreuungssituation oder die Entfernung des Wohnortes der Familie sind für die Art der weiteren Betreuung ebenfalls mitentscheidend. Weiterhin spielen für die Wahl des antibiotischen Regimes nicht nur die lokale Resistenzlage der Erreger, sondern auch die vorausgegangene antineoplastische Therapie sowie individuelle Probleme des Patienten eine große Rolle. Zusätzliche Kenntnisse der durch die antineoplastischen Therapie hervorgerufenen Schwächung der körpereigenen Abwehrsysteme könnten helfen, neue Ansatzpunkte für immunmodulatorische Verfahren zu finden. Klinische und genetische Risikofaktoren müssen ebenso wie Laborparameter, die Niedrig- bzw. Hochrisikopatienten sicher identifizieren sollen, in prospektiven multizentrischen Studien geprüft werden. Fernziel wäre es, die Intensität der antimikrobiellen Prophylaxe entsprechend des individuellen Risikos des Patienten zu steuern sowie nur die Patienten, die tatsächlich Infektionen haben, rasch einer ausreichenden Therapie zuzuführen.

\section{Literatur}

${ }^{1}$ Ammann RA, Hirt A, Luthy AR et al. Predicting bacteremia in children with fever and chemotherapy-induced neutropenia. Pediatr Infect Dis J 2004; 23: 61 - 67

2 Baorto EP, Aquino VM, Mullen CA et al. Clinical parameters associated with low bacteremia risk in 1100 pediatric oncology patients with fever and neutropenia. Cancer 2001; 92: 909-913

3 Bodey GP. Infections in cancer patients. Cancer Treat Rev 1975; 2: $89-128$

${ }^{4}$ Bodey GP, Buckley M, Sathe YS et al. Quantitative relationships between circulating leukocytes and infections in patients with acute leukemia. Ann Intern Med 1966; 64: 328 - 340

${ }^{5}$ Boeckh M, Leisenring W, Riddell SR et al. Late cytomegalovirus disease and mortality in recipients of allogeneic hematopoietic stem cell transplants: importance of viral load and T-cell immunity. Blood 2003; 101: 407-414

${ }^{6}$ Chanock SJ, Pizzo PA. Fever in the neutropenic host. Infect Dis Clin North Am 1996; 10: 777 - 796

${ }^{7}$ Cometta A, Zinner S, de Bock R et al. Piperacillin-tazobactam plus amikacin versus ceftazidime plus amikacin as empiric therapy for fever in granulocytopenic patients with cancer. The International Antimicrobial Therapy Cooperative Group of the European Organization for Research and Treatment of Cancer. Antimicrob Agents Chemother 1995; 39: $445-452$

${ }^{8}$ Creutzig U, Jurgens H, Herold R et al. Concepts of the Society of Paediatric Oncology and Haematology (GPOH) and the German Competence Network in Paediatric Oncology and Haematology for the quality controlled development in paediatric oncology. Klin Padiatr 2004; 216: $379-383$

${ }^{9}$ Creutzig U, Zimmermann M, Reinhardt D et al. Early deaths and treatment-related mortality in children undergoing therapy for acute myeloid leukemia: analysis of the multicenter clinical trials AML-BFM 93 and AML-BFM 98. J Clin Oncol 2004; 22: 4384-4393

${ }^{10}$ Creutzig U, Zimmermann M, Reinhardt D et al. [Analysis of causes of death during intensive chemotherapy according to treatment protocol AML-BFM 93]. Klin Padiatr 2003; 215: 151 - 158 
11 de Bont ES, Vellenga E, Swaanenburg JC et al. Plasma IL-8 and IL-6 levels can be used to define a group with low risk of septicaemia among cancer patients with fever and neutropenia. Br J Haematol 1999; 107: $375-380$

${ }^{12}$ Fijen CA, Bredius RG, Kuijper EJ. Polymorphism of IgG Fc receptors in meningococcal disease [letter]. Ann Intern Med 1993; 119: 636

13 Fleischhack G, Kambeck I, Cipic D et al. Procalcitonin in paediatric cancer patients: its diagnostic relevance is superior to that of C-reactive protein, interleukin 6 , interleukin 8 , soluble interleukin 2 receptor and soluble tumour necrosis factor receptor II. Br J Haematol 2000; 111: $1093-1102$

${ }^{14}$ Flomenberg P, Babbitt J, Drobyski WR et al. Increasing incidence of adenovirus disease in bone marrow transplant recipients. J Infect Dis 1994; 169: $775-781$

15 Grigull L, Schrauder A, Schmitt-Thomssen A et al. Efficacy and safety of G-CSF mobilized granulocyte transfusions in four neutropenic children with sepsis and invasive fungal infection. Infection 2002; 30: $267-271$

${ }^{16}$ Groll AH, Walsh TJ. Uncommon opportunistic fungi: new nosocomial threats. Clin Microbiol Infect 2001; 7 (Suppl 2): 8-24

${ }^{17}$ Hann I, Viscoli C, Paesmans M et al. A comparison of outcome from febrile neutropenic episodes in children compared with adults: results from four EORTC studies. International Antimicrobial Therapy Cooperative Group (IATCG) of the European Organization for Research and Treatment of Cancer (EORTC). Br J Haematol 1997; 99: 580 - 588

18 Klastersky J, Paesmans M, Rubenstein EB et al. The Multinational Association for Supportive Care in Cancer risk index: A multinational scoring system for identifying low-risk febrile neutropenic cancer patients. J Clin Oncol 2000; 18: 3038 - 3051

${ }^{19}$ Lehrnbecher T, Bernig T, Hanisch $\mathrm{M}$ et al. Common genetic variants in the interleukin- 6 and chitotriosidase genes are associated with the risk for severe infection in children undergoing therapy for acute myeloid leukemia. Leukemia 2005; 19: 1745-1750

${ }^{20}$ Lehrnbecher T, Fleischhack G, Hanisch M et al. Circulating levels and promoter polymorphisms of interleukin- 6 and 8 in pediatric cancer patients with fever and neutropenia. Haematologica 2004; 89: $234-236$

${ }^{21}$ Lehrnbecher T, Foster C, Vazquez $\mathrm{N}$ et al. Therapy-induced alterations in host defense in children receiving chemotherapy. J Pediatr Hematol Oncol 1997; 19: 399-417

${ }^{22}$ Lehrnbecher T, Varwig D, Kaiser J et al. Infectious complications in pediatric acute myeloid leukemia: analysis of the prospective multi-institutional clinical trial AML-BFM 93. Leukemia 2004; 18: $72-77$

${ }^{23}$ Lehrnbecher T, Venzon D, deHaas M et al. Assessment of measuring circulating levels of interleukin-6, interleukin-8, C-reactive protein, soluble Fc-gamma receptor type III, and mannose-binding protein in febrile children with cancer and neutropenia. Clin Infect Dis 1999; 29: $414-419$

${ }^{24}$ Lehrnbecher T, Welte K. Haematopoietic growth factors in children with neutropenia. Br J Haematol 2002; 116: 28 - 56

${ }^{25}$ Lex C, Korholz D, Kohlmuller B et al. Infectious complications in children with acute lymphoblastic leukemia and T-cell lymphoma - a rationale for tailored supportive care. Support Care Cancer 2001; 9: $514-521$

${ }^{26}$ Lucas KG, Brown AE, Armstrong D et al. The identification of febrile, neutropenic children with neoplastic disease at low risk for bacteremia and complications of sepsis. Cancer 1996; 77: 791 - 798

${ }^{27}$ Madsen K, Rosenman M, Hui S et al. Value of electronic data for model validation and refinement: bacteremia risk in children with fever and neutropenia. J Pediatr Hematol Oncol 2002; 24: 256-262

${ }^{28}$ Mitchell PLR, Morland B, Stevens MCG et al. Granulocyte colony-stimulating factor in established febrile neutropenia: a randomized study of pediatric patients. J Clin Oncol 1997; 15: $1163-1170$
${ }^{29}$ Oude Nijhuis CS, Daenen SM, Vellenga E et al. Fever and neutropenia in cancer patients: the diagnostic role of cytokines in risk assessment strategies. Crit Rev Oncol Hematol 2002; 44: 163-174

30 Oude Nijhuis M, Daenen W. No antibiotics for selected febrile neutropenic cancer patients might be safe and feasible [abstract]. Proc Am Soc Clin Oncol 2003; 22: 736

${ }^{31}$ Pfaller MA, Jones RN, Doern GV et al. Bacterial pathogens isolated from patients with bloodstream infection: frequencies of occurrence and antimicrobial susceptibility patterns from the SENTRY antimicrobial surveillance program (United States and Canada, 1997). Antimicrob Agents Chemother 1998; 42: $1762-1770$

${ }^{32}$ Pittet D, Wenzel RP. Nosocomial bloodstream infections. Secular trends in rates, mortality, and contribution to total hospital deaths. Arch Intern Med 1995; 155: 1177-1184

33 Pizzo PA. Management of fever in patients with cancer and treatmentinduced neutropenia. N Engl J Med 1993; 328: 1323 - 1332

34 Pizzo PA, Robichaud KJ, Wesley R et al. Fever in the pediatric and young adult patient with cancer. A prospective study of $1001 \mathrm{epi}-$ sodes. Medicine (Baltimore) 1982; 61: 153-165

35 Platonov AE, Kuijper EJ, Vershinina IV et al. Meningococcal disease and polymorphism of FcgammaRIIa (CD32) in late complement component-deficient individuals. Clin Exp Immunol 1998; 111: 97 - 101

${ }^{36}$ Rackoff WR, Gonin R, Robinson C et al. Predicting the risk of bacteremia in children with fever and neutropenia. J Clin Oncol 1996; 14: 919-924

${ }^{37}$ Riikonen P, Saarinen UM, Mäkipernaa A et al. Recombinant human granulocyte-macrophage colony-stimulating factor in the treatment of febrile neutropenia: a double blind placebo-controlled study in children. Pediatr Infect Dis J 1994; 13: 197-202

38 Rocha V, Franco RF, Porcher R et al. Host defense and inflammatory gene polymorphisms are associated with outcomes after HLA-identical sibling bone marrow transplantation. Blood 2002; 100: 3908 3918

${ }^{39}$ Santolaya ME, Cofre J, Beresi V. C-reactive protein: a valuable aid for the management of febrile children with cancer and neutropenia. Clin Infect Dis 1994; 18: 589-595

${ }^{40}$ Schaberg DR, Culver DH, Gaynes RP. Major trends in the microbial etiology of nosocomial infection. Am J Med 1991; 91: 72S-75S

${ }^{41}$ Schellong G, Riepenhausen M. Late effects after therapy of Hodgkin's disease: update 2003/04 on overwhelming post-splenectomy infections and secondary malignancies. Klin Padiatr 2004; 216: 364-369

42 Talcott J, Finberg R, Mayer R et al. The medical course of cancer patients with fever and neutropenia: clinical identification of a low-risk subgroup at presentation. Arch Intern Med 1988; 148: 2561 - 2568

${ }^{43}$ Talcott JA, Siegel RD, Finberg R et al. Risk assessment in cancer patients with fever and neutropenia: A prospective, two-center validation of a prediction rule. J Clin Oncol 1992; 10: 316-322

${ }^{44}$ Talcott JA, Whalen A, Clark J et al. Home antibiotic therapy for low-risk cancer patients with fever and neutropenia: a pilot study of $30 \mathrm{pa}-$ tients based on a validated prediction rule. J Clin Oncol 1994; 12: $107-114$

45 Tunkel AR, Sepkowitz KA. Infections caused by viridans streptococci in patients with neutropenia. Clin Infect Dis 2002; 34: 1524- 1529

${ }^{46}$ Wehl G, Allerberger F, Heitger A et al. Trends in infection morbidity in a pediatric oncology ward, 1986-1995. Med Pediatr Oncol 1999; 32: $336-343$

47 Zinner SH. Changing epidemiology of infections in patients with neutropenia and cancer: emphasis on gram-positive and resistant bacteria. Clin Infect Dis 1999; 29: 490-494 